\title{
Targeting glucose metabolism with 2-deoxy-D-glucose for improving cancer therapy
}

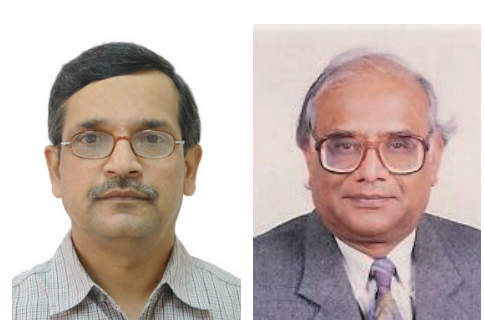

BS Dwarakanath ${ }^{\dagger} \&$ Viney Jain
“Appropriate combinations of tumoricidal agents such as ionizing radiation and chemotherapeutic drugs with 2-DG provide unique opportunities to selectively destroy tumors, reduce toxicity to normal tissues and significantly enhance the therapeutic efficacy."

†Author for correspondence: Institute of Nuclear Medicine \& Allied Sciences, Delhi 110054, India =.Fax:+91 1123918838 =. bsd@inmas.org; bsd@inmas.drdo.in

Tumors require enhanced glucose usage to generate metabolic energy (ATP) and building blocks for macromolecular synthesis in order to sustain rapid cell proliferation. This manifests in increased dependency on the glycolytic pathway (even in the presence of oxygen) for energy (ATP) supply as compared with surrounding normal tissues, a phenomenon first described almost 80 years ago by Otto Warburg [1], who considered this altered metabolic state to be a hallmark of cancer causally associated with the very process of carcinogenesis [2].

The enhanced glucose usage and glycolysis emerging concomitant with malignant transformation, mitochondrial malfunction (linked to or independent of mutations), microregional hypoxia and evolution of aerobic glycolysis in most types of human tumors [3,4] constitute key factors contributing to tumorigenesis and should, therefore, form effective targets for cancer diagnosis, treatment and prevention. While the enhanced glucose uptake is being increasingly exploited for the noninvasive detection and grading of tumors by positron emission tomography using the F-18 labeled glucose analog 2-deoxy-D-glucose (FDG) [5], development of therapeutic strategies based on this metabolic shift still remains a challenge. Recent studies to unravel the intricate relationships between oncogenic transformation, enhanced glucose usage, degree of malignancy and therapeutic resistance of tumors $[3,6]$ have reawakened research interest in the application of Warburg's hypothesis to identify and develop new therapeutic strategies.

A number of pharmacological agents that target glucose transport and/or specific glycolytic enzymes generally overexpressed in tumors are currently under investigation in model systems and in clinical trials. Some promising agents include:

- 2-deoxy-D-glucose (2-DG), inhibitor of glucose transport, phosphohexoisomerase and glucose-6-phosphate dehydrogenase;

- 3-bromopyruvate and lonidamine, inhibitors of hexokinase;

- Inhibitors of pyruvate kinase subtype M2, found mainly in proliferating cells [7-9].

The glucose analog, 2-DG is the most widely investigated pharmacological agent in experimental and clinical oncology for targeting glucose metabolism. 2-DG competitively inhibits glucose transport (by sharing the same glucose transporters) and is phosphorylated by hexokinase (HK) to form 2-DG-6-phosphate, which is not metabolized further, thereby reducing the output from glycolysis (ATP) and the pentose-phosphate pathway (NAPDH) [10-12]. Furthermore, 2-DG alters N-linked glycosylation, leading to unfolded protein responses, and disrupts thiol metabolism, causing oxidative stress. It also induces changes in gene expression and the phosphorylation status of proteins involved in signaling, cell cycle control, DNA repair, calcium influx and cell death via apoptosis [13-15]. Inhibition of proliferation has been reported in many tumor cell lines, while animal studies have revealed a great deal of heterogeneity in the growth inhibition of tumors [16-19].

Initial clinical trials with intravenous infusions of 2-DG in leukemia and pancreatic tumor patients were discontinued due to adverse side effects, mainly in the form of diaphoresis and disturbances of the CNS caused by high doses 
of 2-DG [20]. Recently, Phase I clinical trials in prostrate tumor patients have been initiated following encouraging results in preclinical studies [21]. While the results of these trials are still awaited, the potential of using 2-DG as a single therapeutic agent appears to be limited because of the likelihood of developing toxicity through daily administrations of large doses of 2-DG over a long period of time. The symptoms observed soon after single administration of pharmacological doses of 2-DG are similar to hypoglycemia, mainly due to glucocytopenia in the nervous system (hypothalamus), causing alterations in the regulation of cardio-respiratory and immune systems and other normal tissues [22]. Since 2-DG disappears from the blood with a half-life of approximately $90 \mathrm{~min}$, the symptoms are transitory; however, continuous administration of 2-DG for a long duration could produce serious side effects.

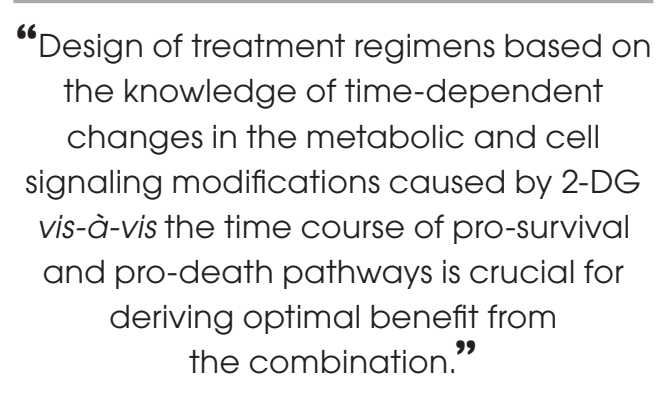

A more promising approach suggested by Jain, Pohlit and Purohit, based on their studies on the energetics of repair of radiation damage in wildtype and respiratory-deficient mutants of yeast cells [23], is to use 2-DG in combination with anticancer agents as a differential modifier of repair processes. This could enhance the efficacies of currently popular therapeutic agents such as radiation and/or cytotoxic drugs [24]. Several in vitro and in vivo studies on various model systems have validated this approach by demonstrating that 2-DG administration before or immediately after irradiation can selectively enhance the induced killing of malignant cells by inhibiting energy-requiring cellular recovery processes involving DNA damage repair and cell cycle check points. The 2-DG-induced enhancement of radiation damage was observed to be directly proportional to the glucose usage, presence of hypoxia and doses of 2-DG and radiation [2426]. It is noteworthy that in normal cell systems under similar conditions, reduction of damage (protection) has been observed [24,27]. While the precise molecular mechanisms underlying the 2-DG-induced differential modifications remain yet to be elucidated, appropriate combinations of tumoricidal agents such as ionizing radiation and chemotherapeutic drugs with 2-DG provide unique opportunities to selectively destroy tumors, reduce toxicity to normal tissues and significantly enhance the therapeutic efficacy.

Based on the 2-DG-induced radiosensitization observed in primary organ cultures of cerebral glioma tissues [28], clinical trials were designed taking into consideration the radiobiology of gliomas and the pharmacokinetics of 2-DG. Since molecular and cellular processes related to survival and death of cells are initiated soon after irradiation and last from several minutes to a few hours (a few days in case of delayed metabolic oxidative stress), the design of treatment regimens based on the knowledge of timedependent changes in the metabolic and cell signaling modifications caused by 2-DG vis$\grave{a}$-vis the time course of pro-survival and prodeath pathways is crucial for deriving optimal benefit from the combination. Preclinical studies using a combination of 2-DG administered intravenously just prior to ( $<5 \mathrm{~min}$ before) irradiation of the tumor showed excellent results, with tumor-free survival rates of $50-60 \%$ $[24,29,30]$. Therefore, the first clinical protocol to test the feasibility and safety consisted of an oral dose of 2-DG (200 mg/kg b.wt) given 20-30 min before $\gamma$-or photon irradiation, with four single weekly fractions of $5 \mathrm{~Gy} /$ fraction [31]. Subsequent dose escalation and multicenter Phase II trials on glioblastoma patients showed excellent compliance, tolerance and safety to the combined treatment of up to a seven-times weekly 2-DG dose of $250 \mathrm{mg} / \mathrm{kg}$ b.wt combined with a $5 \mathrm{~Gy} /$ fraction radiation dose [32]. At a higher dose of $300 \mathrm{mg} / \mathrm{kg}$ b.wt, two out of six patients could not complete treatment due to restlessness, although the vital parameters were not altered [32]. Enhanced survival seen at 2-DG doses of 250-300 mg/kg b.wt suggests that patients who tolerate higher doses would benefit the most from the combined treatment. Most importantly, these trials involving more than 100 glioblastoma patients indicated that the combination of 2-DG with hypofractionated radiotherapy significantly improved the quality of life, with clinicopathological evidence confirming the protection of normal brain tissue [33]. The treatment protocol using weekly large radiation fractions requiring administration of 2-DG only once a week, is convenient for the patient, cost-effective and permits treatment of a larger number of patients per machine. A Phase III multicentric trial is in progress. 
Since radiosensitization by 2-DG increases with radiation dose, a clinical protocol for the treatment of glioblastoma with high-dose stereotactic radiosurgery was designed at the Kettering Medical Center (OH, USA) and approved by the US FDA. A clinical trial using a similar protocol to treat intracranial metastatic lesions is being carried out at the University of Iowa (IA, USA). Results of this trial should provide a basis for the treatment of other inoperable neoplasms with radiosugery plus 2-DG.

The feasibility of using 2-DG in combination with nuclear medicine therapeutic regimens was demonstrated in metastatic thyroid carcinoma patients [24]. Protection of normal bone-marrow cells in whole-body irradiation $[24,27]$ and sensitization of tumor cells to radionuclide-induced damage by 2-DG has been reported [34,35]. These preliminary investigations should encourage larger systematic studies to explore applications of 2-DG for improving the selectivity and efficacy of internal radionuclide therapy, particularly in metastatic diseases. However, optimal design of treatment and dose scheduling should be carefully chosen, as a reduction in the efficacy of radioimmunotherapy using 2-DG was recently reported in a preclinical study [36].

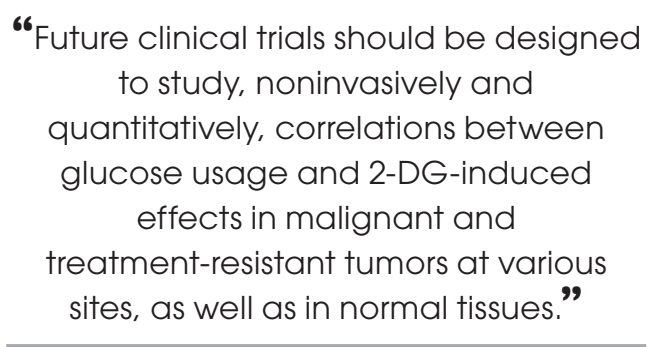

Since cellular responses to damage caused by many anticancer drugs are similar to radiation, 2-DG is also being investigated as an adjuvant in cancer chemotherapy. Although in vitro studies have shown enhanced cell death [37,38], in vivo studies with different anticancer drugs and protocols have demonstrated limited success in obtaining local tumor control [39-40]. Although Phase I clinical trials have begun in the treatment of certain solid tumors [41], further studies are required to optimize treatment schedules for enhancing chemosensitization of tumors by 2 -DG.
To identify patients who would most likely benefit from the combination of 2-DG and radiation or drugs, future clinical trials should be designed to study, noninvasively and quantitatively, correlations between glucose usage and 2-DG-induced effects in malignant and treatment-resistant tumors at various sites, as well as in normal tissues. Attempts should be made to further improve tolerance to higher doses of 2-DG, reduce toxicity and enhance the degree of tumor radiosensitization. Locoregional administration, dietary modifications, relaxation techniques (meditation) or use of sedatives such as diazepam that reduce glucose utilization in the brain could serve to enhance tolerance to 2-DG. Some other novel approaches that deserve to be tested in the clinic include: the use of low doses of 2-DG with the high fat/low carbohydrate ketogenic diet [42]; the combination of 2-DG with inhibitors of the pentose phosphate pathway, such as 6-aminonicotinamide [43,44]; and enhancing tumor glucose usage through the use of tumor-localizing agents such as hematoporphyrin derivatives [45] via intra-tumoral administration.

While research to elucidate precise mechanisms underlying the enhanced glucose metabolism in tumors and its relationship with treatment resistance should be intensified, the success of therapies targeting this phenotype with 2-DG will depend on reducing toxicity to vital organs by optimal design of protocols using novel approaches. For this to happen within a reasonable timespan, in order to make an impact on future oncology, biomedical scientists wellversed with the concepts and tools of molecular genetics, metabolic engineering and systems biology need to work in closer cooperation with clinical oncologists.

\section{Financial \& competing interests disclosure}

The authors have no relevant affliations or financial involvement with any organization or entity with a financial interest in or financial conflict with the subject matter or materials discussed in the manuscript. This includes employment, consultancies, honoraria, stock ownership or options, expert testimony, grants or patents received or pending, or royalties.

No writing assistance was utilized in the production of this manuscript. 


\section{Bibliography}

1. Warburg O: The metabolism of tumors. Constable and Co, London, UK (1930).

2. Warburg O: On the origin of cancer cells. Science 123, 309-314 (1956).

3. Kim J, Dang Chi V: Cancer's molecular sweet tooth and the Warburg effect. Cancer Res. 66, 8927-8930 (2006).

4. Gillies RJ, Robey I, Gatenby RA: Causes and consequences of increased glucose metabolism of cancers. J. Nucl. Med. 49, S24-S42 (2008).

5. Gambhir SS: Molecular imaging of cancer with positron emission tomography. Nat. Rev. Cancer 2, 683-693 (2002).

6. Cao W, Fang F, Gibbs $S$ et al:: Glucose uptake inhibitor sensitizes cancer cells to daunorubicin and overcomes drug resistance in hypoxia. Cancer Chemother. Pharmacol. 59, 495-505 (2007).

7. Xu RH, Pelicano H, Zhou Y et al.: Inhibition of glycolysis in cancer cells: a novel strategy to overcome drug resistance associated with mitochondrial respiratory defect and hypoxia. Cancer Res. 65, 613-621 (2005).

8. Di Cosimo S, Ferretti G, Papaldo P et al.: Lonidamine: Efficacy and safety in clinical trials for the treatment of solid tumors. Drugs Today 39, 157-173 (2003).

9. Spoden GA, Mazurek S, Morandell D et al: Isotype-specific inhibitors of the glycolytic key regulator pyruvate kinase subtype M2 moderately decelerate tumor cell proliferation. Int. J. Cancer 123, 312-321 (2008).

10. Woodward GE, Cramer FB: 2-deoxy-Dglucose as an inhibitor of anaerobic glycolysis in tumor tissues. J. Franklin. Inst. 254, 259-260 (1952).

11. Brown J: Effects of 2-deoxyglucose on carbohydrate metabolism: review of the literature and studies in the rat. Metabolism 11, 1098-1112 (1962).

12. McComb RB, Yushok WD: Metabolism of ascites tumor cells. IV. Enzymatic reactions involved in adenosinetriphosphate degradation induced by 2-deoxyglucose. Cancer Res. 24, 198-205 (1964).

13. Lin X, Zhang F, Bradbury CM et al.: 2-Deoxy-D-glucose-induced cytotoxicity and radiosensitization in tumor cells is mediated via disruptions in thiol metabolism. Cancer Res. 63, 3413-3417 (2003).

14. Kurtoglu M, Gao N, Shang J et al:: Under normoxia, 2-deoxy-D-glucose elicits cell death in select tumor types not by inhibition of glycolysis but by interfering with $\mathrm{N}$-linked glycosylation. Mol. Cancer Ther. 6, 3049-3058 (2007).

15. Heminger K, Jain V, Kadakia M, Dwarakanath B, Berberich SJ: Altered gene expression induced by ionizing radiation and glycolytic inhibitor 2-deoxy-glucose in a human glioma cell line: implications for radiosensitization. Cancer Biol. Ther. 5 , 815-823 (2006).

16. Bell SE, Quinn DM, Kellett GL, Warr JR: 2-deoxy-D-glucose preferentially kills multidrug-resistant human $\mathrm{KB}$ carcinoma cell lines by apoptosis Br. J. Cancer 78, 1464-1470 (1998).

17. Dwarakanath BS, Adhikari JS, Khaitan D et al:: Growth inhibition and induction of apoptosis by 2-deoxy-D-glucose in human squamous carcinoma cell lines. Biomedicine 24, 36-47 (2005).

18. Laszlo J, Humphrey SR, Glodin A: Effects of glucose analogues (2-deoxy-D-glucose, 2-deoxy-D-galactose) on experimental tumors. J. Natl Cancer Inst. 24, 267-271 (1960).

19. Hunter AJ, Blekkenhorst GH: The effects of 2-deoxyglucose and amino-oxyacetic acid on the radiation response of mammalian cells in vitro. Int. J. Radiat. Biol. 73, 311-324 (1998).

20. Landau BR, Laszlo J, Stengle J et al.: Certain metabolic and pharmacologic effects in cancer patients given infusions of 2-deoxy-D-glucose. J. Nat. Cancer Inst. 21, 485-494 (1958).

21. Diaola RS, Dvorzhinski D, Thalasia A et al.: Therapeutic starvation and autophagy in prostate cancer: a new paradigm for targeting metabolism in cancer therapy. Prostate 68, 1743-1752 (2008).

22. Vijayaraghavan R, Kumar D, Dube SN et al.: Acute toxicity and cardio-respiratory effects of 2-deoxy-D-glucose: a promising radiosensitizer. Biomed. Environm. Sci. 19, 96-103 (2006).

23. Jain V, Pohlit W, Purohit SC: Influence of energy metabolism on the repair of X-ray damage in living cells. III. Effects of 2-deoxy$\mathrm{D}$-glucose on liquid holding reactivation in yeast. Biophysik. 10, 137-142 (1973).

24. Jain V: Modifications of radiation responses by 2-deoxy-D-glucose in normal and cancer cells. Ind. J. Nucl. Med. 11, 8-17 (1996).

25. Dwarakanath BS, Jain VK: Energy linked modification of the radiation response in a human cerebral glioma derived cell line. Int. J. Radiat. Oncol. Biol. Phys. 17, 1033-1040 (1989).

26. Dwarakanath BS, Zolzer F, Chandna S et al: : Heterogeneity in 2-deoxy-D-glucose induced modifications in energetic and radiation responses of human tumor cell lines. Int. J. Radiat. Oncol. Biol. Phys. 51, 1151-1161 (2001).

27. Jain VK, Kalia VK, Gopinath PM, Naqvi S, Kucheria K: Optimization of cancer therapy, part III. Effects of combinig 2-deoxy-D-glucose treatment with $\gamma$ irradiation on normal mice. Ind. J. Exp. Biol. 17, 1320-1325 (1979).
28. Dwarakanath BS, Jain VK: Modification of the radiation induced damage by 2 -deoxy-Dglucose in organ cultures of human cerebral gliomas. Int. J. Radiat. Oncol. Biol. Phys. 13, 741-746 (1987).

29. Juling-Pohlit L, Pohlit W, Blattmann H: Repair inhibition in tumors irradiated with fast protons and negative pions. Strablenther. Onkol. 166, 6-9 (1990).

30. Dwarakanath BS, Singh S, Jain V: Optimization of tumour radiotherapy: Part $\mathrm{V}$ - radiosensitization by 2 -deoxy-D-glucose and DNA ligand Hoechest-33342 in a murine tumour. Ind. J. Exp. Biol. 37, 865-870 (1999).

31. Mohanti BK, Rath GK, Anantha N et al.: Improving cancer radiotherapy with 2-deoxy-D-glucose: Phase I/II clinical trials on human cerebral gliomas. Int. J. Radiat. Oncol. Biol. Phys. 35, 103-111 (1996).

32. Singh D, Banerji AK, Dwarakanath BS et al.: Optimizing cancer radiotherapy with 2-deoxy-D-glucose: dose escalation studies in patients with glioblastoma multiforme. Strablentherapie 181, 507-514 (2005).

33. Venkataramana NK, Prasanna KV, Vishwanath PN et al: : Enhanced radiation effect on the tumor with protection of normal brain during a combinational therapy of 2-deoxy-D-glucose and hypo fractionated radiotherapy in malignant gliomas. Presented at: International Symposium on Application of 2-Deoxy-D-Glucose in the Management of Cancer. INMAS, Delhi, India, November 8-10, 2006.

34. Shrivastava V, Mishra AK, Dwarakanath BS, Ravindranath T: Enhancement of radionuclide induced cytotoxicity by 2-DG in human tumor cell lines. J. Cancer Res. Ther. 2, 57-64 (2006).

35. Aft RL, Lewis JS, Zhang F, Kim J, Welch MJ: Enhancing targeted radiotherapy by copper (II) diacetyl-bis(N4methylthiosemicarbazone) using 2-deoxy-D-glucose. Cancer Res. 63, 5496-5504 (2003).

36. Jason L, Dearling J, Qureshi U et al.: Combining radioimmunotherapy with antihypoxia therapy 2-deoxy-D-glucose results in reduction of therapeutic efficacy. Clin. Cancer Res. 13, 1903-1910 (2007).

37. Dwarakanath BS, Khaitan D, Ravindranath T: 2-deoxy-D-glucose enhance the cytotoxic effects of topoisomerase inhibitors in human tumor cell lines. Cancer Biol. Ther. 3, 34-43 (2004).

38. Simons AL, Ahmad IM, Mattson DM, Dornfeld KJ, Spitz DR: 2-deoxy-D-glucose combined with cisplatin enhances cytotoxicity via metabolic oxidative stress in human head and neck cancer cells. Cancer Res. 67, 3364-3370 (2007). 
39. Maschek G, Savaraj N, Priebe W et al.: 2-deoxy-D-glucose increases the efficacy of adriamycin and paclitaxel in human osteosarcoma and non-small cell lung cancers in vivo. Cancer Res. 64, 31-34 (2004).

40. Gupta S, Mathur R, Dwarakanath BS: Modifications of etoposide induced toxicity by 2-deoxy-D-glucose in Ehrlich ascites tumor bearing mice. Cancer Biol. Ther. 4, 87-94 (2005).

41. Raez LE, Rosenblatt J, Schlesselman J et al.: Combining glycolytic inhibitors with chemotherapy: Phase I trial of 2-deoxyglucose and docetaxel in patients with solid tumors. Presented at: American Society of Clinical Oncology Annual Meeting, Orlando, FL, USA, May 13-17 (2005).
42. Marsh J, Mukherjee P, Seyfried TN: Drug/diet synergy for managing malignant astrocytoma in mice: 2-deoxy-D-glucose and the restricted ketogenic diet. Nutr. Metab. 5, 33-37 (2008).

43. Varshney R, Dwarakanath BS, Jain V: Radiosensitization by 6 -aminonicotinamide and 2-deoxy-D-glucose in human cancer cells. Int. J. Radiat. Biol. 81, 397-408 (2005).

44. Varshney R, Gupta S, Dwarakanath BS: Radiosensitization of murine Ehrlich Ascites tumor by a combination of 2-deoxy-D-glucose and 6 amino nicotinamide. Tech. Cancer Res. Treat. 3, 659-663 (2004).

45. Dwarakanath BS, Adhikari JS, Jain V: Hematoporphyrin derivatives potentiate the radiosensitizing effects of 2-DG in cancer cells. Int. J. Radiat. Oncol. Biol. Phys. 43, 1125-1133 (1999).

\section{Affiliations}

- BS Dwarakanath

Institute of Nuclear Medicine \& Allied Sciences, Delhi 110054, India

Fax: +91 1123918838

bsd@inmas.org; bsd@inmas.drdo.in

- Viney Jain

Eco-Development Foundation, New Delhi 110001, India Tel.: +91 1244070885

vineyjain@gmail.com 